\title{
Endovascular revascularization of chronically occluded vertebral artery: single-center experience
}

\author{
Xueli Cai ${ }^{1,2}$, Liangtong Huang ${ }^{2}$, Xueping Chen², Jingping Sun ${ }^{2}$, Feng Gao ${ }^{1}$ \\ ${ }^{1}$ Department of Neurology, The Second Afliated Hospital, Zhejiang University, School of Medicine, Hangzhou, Zhejiang, China \\ ${ }^{2}$ Department of Neurology, Lishui Hospital of Zhejiang University (Lishui Municipal Central Hospital), Lishui, Zhejiang, China
}

\begin{abstract}
Introduction: Chronic vertebral basilar artery occlusion is one of the most common causes of ischemic stroke, which accounts for roughly $20 \%$ of all cases. However, the evidence for the precise clinical effect in treatment of the initial segment of chronic vertebral basilar artery occlusion is not sufficient.

Aim: To evaluate the feasibility and efficacy of endovascular therapy in the initial segment of chronic vertebral basilar artery occlusion.

Material and methods: This is a retrospective study based on data obtained from Lishui Hospital of Zhejiang University. We identified patients who underwent treatment for initial segment occlusion of the vertebral basilar artery from September 2014 to September 2018.

Results: Among a total of twenty-two subjects, $77.27 \%$ of them were men and the median age was 61 years old. The primary medical history of these patients included hypertension $(15,68.18 \%)$, hyperlipidemia (13, 59.09\%), diabetes mellitus (11,50.00\%), and coronary artery disease (6, 27.27\%). About 54.54\% of the patients were current smokers. Successful recanalization was achieved in $86.36 \%$ of patients. Five cases of balloon dilatation were recanalized and fourteen cases were treated by balloon dilation combined with stent implantation. During the follow-up period, there were no recurrent cerebral ischemic events, including transient ischemic attack or stroke. Six (27.27\%) arteries were found to exhibit angiographic re-stenosis.

Conclusions: The clinical effect of balloon dilatation or balloon dilatation combined with stenting in the treatment of chronic vertebral basilar artery occlusion in the beginning segment was feasible and safe.
\end{abstract}

Key words: endovascular therapy, clinical effect, chronic vertebral basilar artery occlusion.

\section{Introduction}

Ischemic stroke accounts for about $80 \%$ of strokes, while ischemic stroke, caused by posterior circulation ischemia, accounts for about $20 \%$ of cases [1, 2]. As with the anterior circulation, the main cause of ischemia in the posterior circulation is atherosclerosis, and the starting segment of the vertebral artery and the intracranial segment are the most common sites of atherosclerosis [3]. The incidence of chronic vertebral artery occlusion in the Chinese population is higher than that of Caucasian people [4]. The results of the posterior circulation enrollment study in New England Medical Center (NEMC-PCR) showed that roughly one-third of cases of posterior circulatory stroke involved narrowness or occlusion of the initial segment of the vertebral artery [5]. In patients with posterior circulation ischemia, the incidence of

Address for correspondence

Feng Gao, Department of Neurology, The Second Afliated Hospital, Zhejiang University School of Medicine, 88 JieFang St, Hangzhou

310009, Zhejiang, China, e-mail: 2202012@zju.edu.cn; Jingping Sun, Department of Neurology, Lishui Hospital of Zhejiang University

(Lishui Municipal Central Hospital), 289 KuoCang St, LiShui 323000, Zhejiang, China, e-mail: beautysjpyz@163.com 
significant narrowing (50\% stenosis) or occlusion at the origin of the vertebral artery was $32.1 \%$; the incidence of significant stenosis or occlusion in the intracranial segment of the vertebral artery was $32.4 \%$; and the incidence of vertebral artery stenosis or occlusion was very high in patients with posterior circulation ischemia [3]. However, the studies reporting chronic occlusion of the vertebral basilar arteries were limited and thus the optimal treatments remains undefined. Although in some patients, blood perfusion can be maintained through compensation by collateral vessels, for some patients this is not enough, which results in insufficient blood perfusion to certain parts of the brain. Previous studies have shown that patients with chronic vertebral artery occlusion, who have poor drug treatment outcomes, have a significantly increased risk of developing posterior circulation ischemia [6]. In recent years, owing to the development of new specialized neuro-interventional techniques and surgical instruments, studies reporting recanalization of chronic intracranial and extracranial aortic occlusion have gradually increased. In China and abroad, however, there have only been a few studies that report recanalization of the vertebral artery due to its chronic occlusion at its base. In our study, we analyzed and summarized the procedures completed in the past 5 years, that focused on vertebral-basilar recanalization for chronic occlusions that affected the initial segment of the vessel. We also explored the feasibility, safety and clinical efficacy of this technique.

\section{Aim}

In the present study, we retrospectively reviewed 22 cases of chronically occluded vertebral arteries, treated with balloon dilatation or balloon-expandable stent angioplasty. Our main objective was to evaluate the feasibility and efficacy of endovascular therapy in the treatment of the initial segment of chronic vertebral basilar artery occlusion. The main endpoints were residual stenosis, improvement of symptoms and complication occurrence.

\section{Material and methods}

\section{Study population}

This is a retrospective study based on data obtained from our stroke intervention database from the department of neurology, Lishui Hospital of Zhejiang University. We identified patients who under- went treatment for initial segment chronic occlusion of the vertebral basilar artery from September 2014 to September 2018. "Chronic occlusions" were defined as symptomatic (transient ischemic attack TIA or stroke), complete initial segment, occlusion of the vertebral basilar artery that was presumably caused by atherosclerosis, and in which endovascular therapy was performed after 1 month from the time last seen and clearly diagnosed by magnetic resonance angiography (MRA), digital subtraction angiography (DSA) $[7,8]$. Studies were selected according to the following inclusion criteria: (1) occlusion of the initial segment of the vertebral artery, occlusion should be confirmed by DSA; (2) patients with repeated dizziness, vertigo, ataxia, diplopia, nausea, vomiting or dysarthria; (3) If the vertebral artery is occluded, the occlusion segment should occur on the dominant side or bilateral side; (4) clinical symptoms not improved after conservative treatment through internal medicine for more than 3 months; (5) excluding surgical contraindications.

This research protocol was approved by the Ethics Committee of the Lishui Hospital of Zhejiang University.

\section{Preprocedural medical management}

All the patients underwent preprocedural routine examinations and DSA, in which we especially focused on bilateral vertebral angiography to determine occluded blood vessels with responsible lesions. Routine examinations were also performed to understand the condition of collateral blood circulation. In the meantime, all patients were prescribed (Bayer, Leverkusen, Germany) 100 mg/day aspirin and clopidogrel (Plavix, Sanofi Aventis, France) $75 \mathrm{mg} /$ day at least 3 days before intervention. Atorvastatin $20 \mathrm{mg} /$ day (Pfizer, Dalian, China) was also routinely given before surgery.

\section{Intervention technique}

The endovascular procedures were performed under local anesthesia; the right femoral artery was punctured by the Seldinger method using a $6 \mathrm{~F}$ guiding catheter (Cordis Corporation, Miami Lakes, USA). Intravenous heparin boluses were given at $0.75 \mathrm{mg} / \mathrm{kg}$ body weight to maintain the activated clotting time between $250 \mathrm{~s}$ and $300 \mathrm{~s}$ during the procedure. We conducted a comprehensive assessment of the vessel pathway prior to intervention. Under the guidance of 
the route, the micro guidewire (Stryker, Kalamazoo, Michigan, USA) and the microcatheter (Micro Therapeutics Inc. dba ev3 Neurovascular, Irvine, USA) were carefully probed and entered the true vascular cavity through the occluded segment. Subsequently, microcatheter angiography was used to confirm that the distal end of the catheter was located in the true lumen of the vessel, and also to assess the degree of blood circulation to the distal branch. At the same time, the length of the blocked segment was measured. All lesions were initially predilated with a conventional balloon segment by segment. Angiography was performed to confirm whether the vessel was recanalized. Then, the suitable balloon-expandable stent (3-4 mm × 12-18 mm, Apoll 4008, MicroPort, Z.JHigh-Tech Park Shanghai, China) was placed in the occlusion within the vertebral artery guided by a micro guidewire. At this point, the stent was directly released when the expansion pressure reached 6-12 atm. After release and slow withdrawal of the balloon, we again used angiogram imagery to re-evaluate the lumen to make sure the occlusion recanalized; we also did this to exclude vessel perforation, dissection, or distal embolization. The recanalization was considered a success if the residual stenosis of the occluded segment was less than $20 \%$. The patients were closely observed for 3 days after surgery, and routine use of clopidogrel, combined with aspirin, was prescribed for 6 months.

\section{Postprocedural management}

After the procedure, patients with the arterial sheath were routinely sent to the ward for continuous observation and blood pressure monitoring. Intravenous low molecular weight heparin boluses were given subcutaneously for once every $12 \mathrm{~h}$ for 3 consecutive days. Subsequently, the femoral artery sheath was removed after $4 \mathrm{~h}$ of heparin natural neutralization. Dual antiplatelet therapy comprising aspirin $100 \mathrm{mg}$ and clopidogrel $75 \mathrm{mg}$ was continued for 6 months after the procedure. Then according to the results of the reexamination, the aspirin tablets were taken for a long time.

\section{Data collection and follow-up outcome}

All patients were followed up clinically at 3 months, 6 months and 12 months. They were scheduled to return for a vascular imaging examination (CTA or DSA examination) at 3 months, 6 months and
12 months. Demographic, clinical features, angiographic, and procedural data were collected. The clinical outcome was judged by modify Rankin Scale (mRS) and the vascular restenosis condition. Restenosis was defined as a diameter of the stenosis within $3 \mathrm{~mm}$ of the stent, or if the stenosis in the stent was $>50 \%$ or the degree of stenosis increased by $30 \%$. The measurement method adopted the WASID standard.

\section{Statistical analysis}

Descriptive statistics were used in this study. Continuous data are expressed as mean \pm standard deviation (SD) or as the median with interquartile range (IQR), whereas categorical data were presented as numbers and percentages. Statistical analyses were performed using the SPSS software (SPSS Inc., Chicago, USA).

\section{Results}

\section{Patient demographics}

A total of 22 subjects were collected following the inclusion criteria used by computed tomography angiography (CTA). $77.27 \%$ were men, and the others were female. The median (IQR) age was 61 (38-85) years old. The primary medical history of these patients included hypertension $(15,68.18 \%)$, hyperlipidemia $(13,59.09 \%)$, diabetes mellitus (11, $50.00 \%)$, and coronary artery disease $(6,27.27 \%)$. Moreover, $54.54 \%$ of patients were current smokers. The median time taken to perform the operation from symptom onset was 2.6 weeks (Table I).

\section{Angiographic and procedural characteristics}

Successful recanalization was achieved in $86.36 \%$ of patients (19 cases). An illustrative case is shown in Photos 1 and 2. Five of the total patients were treated with balloon angioplasty only. Fourteen (73.68\%) were treated with angioplasty followed by stenting. The intra-procedural complications included dissection during lesion traversal, which occurred in $4.55 \%$ of patients (1 case). No perforation, hyperperfusion syndrome, distal embolization, or other device-related complications occurred during the procedure. The details are listed in Table II.

\section{In-hospital and follow-up outcomes}

As shown in Table III, no patients presented with a new minor ischemic stroke after the procedure 
Table I. Patients' baseline demographic and clinical characteristics $(N=22)$

\begin{tabular}{|c|c|}
\hline Characteristics & $N(\%)$ or median (IQR) \\
\hline $\begin{array}{l}\text { Symptom onset to operation, } \\
\text { median }(\mathrm{IQR})[\text { months] }\end{array}$ & $2.6(1.6-7.1)$ \\
\hline Age, median (IQR) [years] & $61(38-85)$ \\
\hline Male & $17(77.27 \%)$ \\
\hline \multicolumn{2}{|l|}{ Medical history: } \\
\hline Hypertension & $15(68.18 \%)$ \\
\hline Hyperlipidemia & $13(59.09 \%)$ \\
\hline Diabetes mellitus & $11(50.00 \%)$ \\
\hline Cardiovascular disease & $6(27.27 \%)$ \\
\hline Dyslipidemia & $13(59.09 \%)$ \\
\hline Smoking & $12(54.54 \%)$ \\
\hline \multicolumn{2}{|l|}{ Clinical: } \\
\hline Preoperative mRS, median (IQR) & $2.0(1.0-3.0)$ \\
\hline $\begin{array}{l}\text { Symptom onset to treatment, } \\
\text { median (IQR) [weeks] }\end{array}$ & $2.6(1.6-7.1)$ \\
\hline $\begin{array}{l}\text { Occlusion confirmed to treat- } \\
\text { ment, median (IQR) [weeks] }\end{array}$ & $1.2(0.6-3.8)$ \\
\hline
\end{tabular}

and no deaths were reported. During the clinical follow-up period, there were no recurrent cerebral ischemic events, including TIA or stroke. Six (27.27\%) arteries were found to exhibit angiographic restenosis. Owing to the fact that no recurrent symptoms could be documented, the artery was temporarily left without further vessel revascularization.

\section{Discussion}

The clinical manifestations of vertebral basilar artery occlusion can vary from person to person; a patient may suffer from transient symptoms or a fatal stroke. Clinically, the symptoms of acute vertebral basilar artery occlusion are relatively severe and the prognosis is poor. There have been some studies on the treatment of related diseases, and some surgical methods and effects have gradually gained recognition $[9,10]$. However, regarding the study of chronic vertebral artery occlusion, only a few studies and reports have been published. Currently, the limited number of studies have predominantly focused on the V4 segment of the vertebral artery [11]. However, there still have been no reports on the occlusion of the initial segment of the vertebral artery.

Although chronic occlusion of the vertebral artery causes a less disabling stroke, it often causes a series of symptoms of posterior circulation ischemia, such as dizziness, weakness, speech disorder, blurred vision, ataxia, and mental disorders. Clinical drug treatment often yields poor results, which seriously affects the quality of life of the affected patients. In recent years, endovascular treatment for chronic vertebral artery occlusion at the beginning segment has gradually been carried out. A previous study showed that the related cases of chronic vertebral artery occlusion have a high recanalization rate, but the rate of complications during the operation was also higher, and was significantly higher than that of intracranial and extravascular stenosis [7]. Dashti et al. [8] reported that 9 patients had perioperative complications, including 2 deaths. In recent years, due to the improvement of surgical methods, materials and preoperative evaluation, the incidence of related complications has decreased. Furthermore, the feasibility and safety of the recanalization of chronic vertebral artery occlusion have been gradually recognized. We preliminarily summarized the indications for the interventional treatment of chronic vertebral artery occlusion at the beginning segment: (1) there was symptomatic stroke or TIA related to occlusion, and drug treatment was ineffective; (2) preoperative imaging showed hypoperfusion of the area supplied by the vertebral basilar artery; (3) no serious neurological dysfunction and/ or massive cerebral infarction.

In our surgery, we discovered that the most critical and difficult part of incising and opening the stenosis involved the process of passing the guide wire through the anatomical occlusion segment to reach the blood vessels' true lumen. It is more difficult to find the occlusion stump and reach the true lumen of the blood vessel if the occlusion is at the initial segment of the vertebral artery. For this reason, it is important for us to have extensive surgical experience and perform an adequate pre-surgical evaluation. We aim to carefully assess the length of the occlusion segment, as well as the vascular anatomy of the occlusion segment, before initiating the surgery. After that, we evaluate fully the patient's bilateral posterior circulation vessels, through conventional head and neck CTA and DSA. By using CTA and DSA we are able to appreciate the vascular length of 

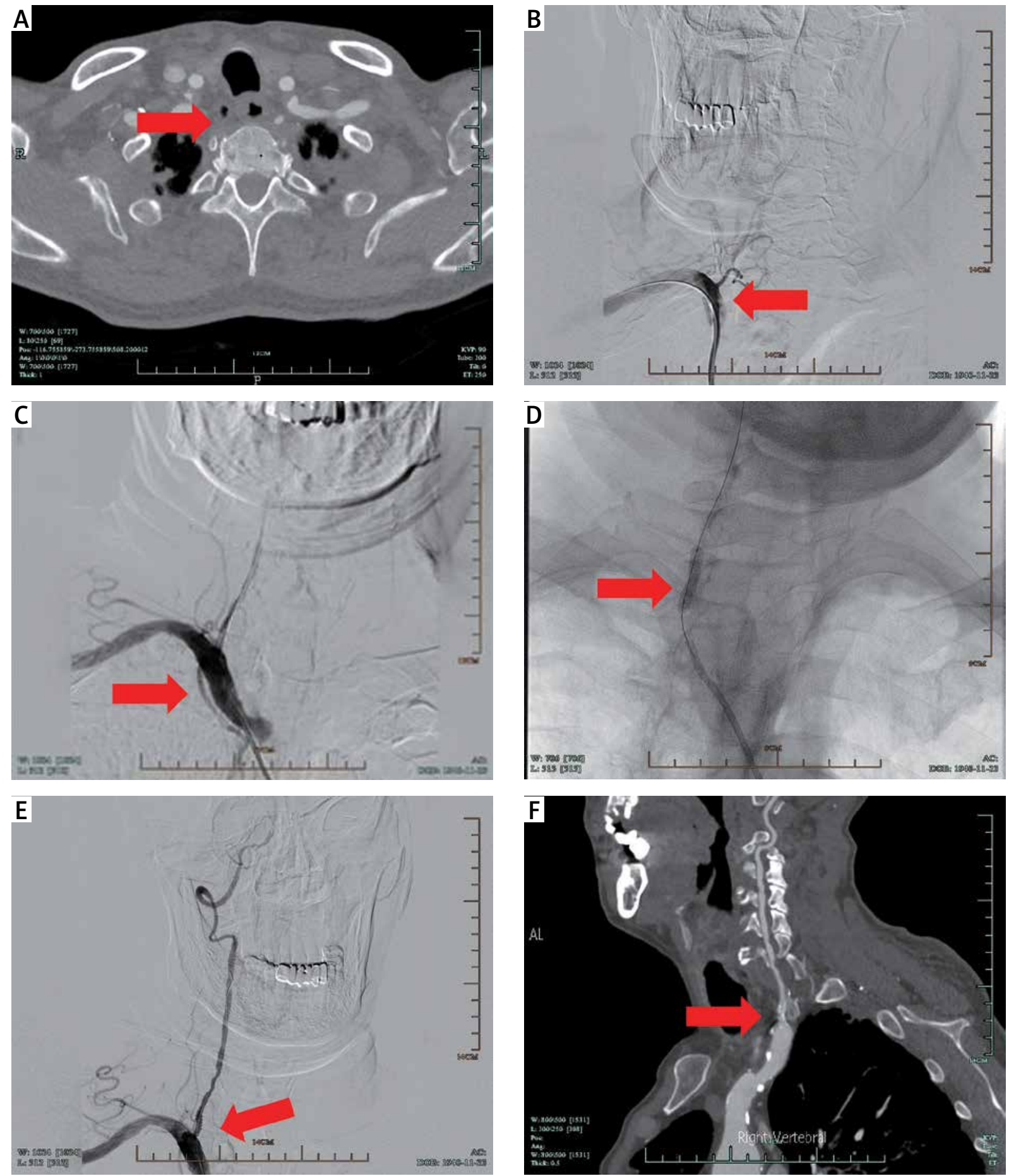

Photo 1. Example of balloon-expanded stent treatment for vertebral basilar artery occlusion at the initial segment and follow-up. A - The preoperative CTA revealed severe right vertebral artery stenosis at the initial segment. B - The preoperative DSA illustrated right vertebral artery stenosis at the initial segment. $\mathbf{C}$ - The micro guidewire passes through the segment of occlusion. $\mathbf{D}$ - The DSA showed that the balloon gradually dilated the occluded segment. E-Angiographic result after the procedure with balloon-expanded stents showed good antegrade perfusion. F - CTA result postoperatively showed no restenosis in the vertebral artery after 6 months of follow-up (as the arrow shows in A-F) 

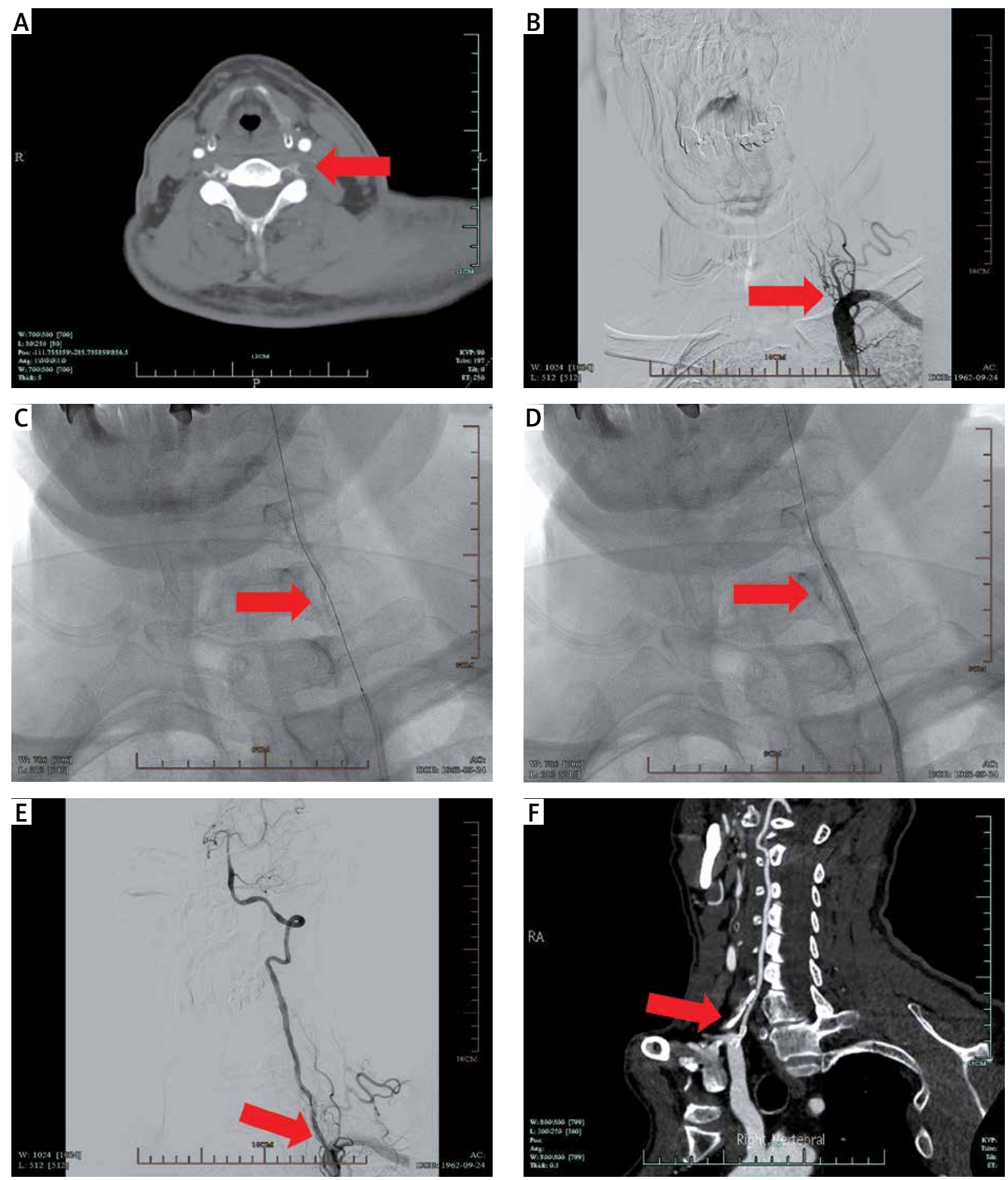

Photo 2. Example of balloon-expanded stent treatment for vertebral basilar artery occlusion at the beginning segment and follow-up. A - The preoperative CTA revealed severe right vertebral artery stenosis at the beginning segment. B - The preoperative DSA illustrated right vertebral artery stenosis at the beginning segment. $\mathbf{C}$ - The micro guidewire passes through the segment of occlusion. D - The DSA showed that the balloon gradually dilated the occluded segment. E - Angiographic result after the procedure with balloon-expanded stents showed good antegrade perfusion. F - CTA result postoperatively showed no restenosis in the vertebral artery at the 6-month follow-up (as the arrow shows in A-F) 
Table II. Angiographic and procedural characteristics

\begin{tabular}{|lc|}
\hline Characteristics & $N(\%)$ or median \\
\hline Outcome: & $19(86.36 \%)$ \\
\hline Successful recanalization & $3(13.64 \%)$ \\
\hline Residual stenosis & $5(26.32 \%)$ \\
\hline Angioplasty & $14(73.68 \%)$ \\
\hline Stenting & $1(4.55 \%)$ \\
\hline Complication rate: & $0(0.00 \%)$ \\
\hline $\begin{array}{l}\text { Dissection } \\
\text { Symptomation }\end{array}$ & $0(0.00 \%)$ \\
\hline Hyperperfusion syndrome & $0(0.00 \%)$ \\
\hline Branch embolization & $0(0.00 \%)$ \\
\hline
\end{tabular}

Successful recanalization: residual stenosis of occluded segment $<20 \%$ at the end of the intervention. Residual stenosis: residual stenosis of occluded segment $\geq 20 \%$ stenosis at the end of the intervention.

the occlusion segment, in addition to the structural anatomy of the occlusion segment. We aim to fully anticipate any surgical challenges or compilations before initiating the operation. Our surgeons have nearly 10 years of experience in intracranial vascular surgery, micro-wire manipulation, intraoperative multi-angle angiography, as well as road maps. These are the most critical areas of expertise that are required for successful surgery. In addition, the pre-judgment and management of intraoperative complications are also critical to surgery.

In this study, 22 patients were enrolled. The occluded vascular condition was observed by DSA before starting the operation. The vascular opening was successful in 19 patients, and the opening rate was $86.36 \%$. All 3 patients underwent intraoperative attempts, but all attempts to pass the guide wire beyond the lesions were unsuccessful. In 1 case intraoperative intercalation occurred. After administering antiplatelet treatment, CTA reexamination was performed. No other serious surgical complications occurred. Compared with their preoperative state, after recanalization, most patients showed various degrees of improvement in symptoms and a decline in mRS when followed up. Furthermore, most patients were satisfied with the outcome of the operation. Those whose large blood vessels experience lateral
Table III. Clinical and imaging outcomes in hospital and during follow-up

\begin{tabular}{|lc|}
\hline Characteristics & $N$ (\%) or median \\
\hline Outcome: & $22(100.00 \%)$ \\
\hline In-hospital & $0(0.00 \%)$ \\
\hline Deatroke & $0(0.00 \%)$ \\
\hline \begin{tabular}{l} 
Follow-up: \\
\hline Deatroke/TIA
\end{tabular} & $0(0.00 \%)$ \\
\hline $\begin{array}{l}\text { mRS score at the 6 } \\
\text { median (IQR) }\end{array}$ & $0(0.00 \%)$ \\
\hline $\begin{array}{l}\text { Imaging follow-up time } \\
(n=22, \text { months) }\end{array}$ & $1(0-3)$ \\
\hline \begin{tabular}{l} 
Angiographic restenosis \\
\hline Symptomatic restenosis
\end{tabular} & $5.6(3.6-10.3)$ \\
\hline
\end{tabular}

TIA - transient ischemic attack.

branch compensation did not suffer from large-area cerebral infarction during severe perfusion deficiency. Also, after surgery, the perfusion was ameliorated and the quality of the patients' life improved.

Our study shows that this procedure is a relatively safe and effective method of treatment. Failure of recanalization is common in chronic occlusion of the vertebral artery. This failure may be related to calcification, density or length of the occlusion segment. In 1 patient in this study, all attempts to pass the occlusion lesion were unsuccessful. In cases similar to this situation, a more aggressive attempt may have serious complications. In-stent restenosis is another challenge that we face during intravascular surgery. We followed up 6 cases of vascular restenosis that were considered to be associated with higher postoperative residual stenosis and longer lesions [12]. Some studies have found that stent restenosis can be treated by balloon angioplasty or repeated stenting in symptomatic patients [13]. However, regarding our patients with restenosis, we did not perform further interventions because our patients remained symptomless.

Our research does have several limitations. Since this is a retrospective, single-center study, with a small sample size, further multicenter prospective studies are needed to confirm our findings. None- 
theless, our study showed a satisfactory rate of open vertebral artery occlusion and a very low incidence of complications.

\section{Conclusions}

Our study illustrated the experiences and the clinical effect of balloon dilatation or balloon dilatation combined with stenting in the treatment of chronic occlusion of the vertebral artery, which was feasible and safe.

\section{Acknowledgments}

This work was supported by grants from the Lishui Science and Technology Project (No. 2018RC22), Training for High-level Talents Program of Lishui in the Health Commission of Zhejiang Province.

\section{Conflict of interest}

The authors declare no conflict of interest.

\section{References}

1. Writing GM, Mozaffarian D, Benjamin EJ, et al. Heart Disease and Stroke Statistics-2016 Update: a report from the American Heart Association. Circulation 2016; 133: e38-360.

2. Savitz SI, Caplan LR. Vertebrobasilar disease. N Engl J Med 2005; 352: 2618-26.

3. Caplan L, Wityk R, Pazdera L, et al. New England Medical Center Posterior Circulation Stroke Registry II. Vascular Lesions. J Clin Neurol 2005; 1: 31-49.

4. Wong LK. Global burden of intracranial atherosclerosis. Int J Stroke 2006; 1: 158-9.

5. Wityk RJ, Chang HM, Rosengart A, et al. Proximal extracranial vertebral artery disease in the New England Medical Center Posterior Circulation Registry. Arch Neurol 1998; 55: 470-8.

6. Lindsberg PJ, Soinne L, Roine RO, et al. Options for recanalization therapy in basilar artery occlusion. Stroke 2005; 36: 203-4.

7. Lin R, Aleu A, Jankowitz B, et al. Endovascular revascularization of chronic symptomatic vertebrobasilar occlusion. J Neuroimaging 2012; 22: 74-9.

8. Dashti SR, Park MS, Stiefel MF, et al. Endovascular recanalization of the subacute to chronically occluded basilar artery: initial experience and technical considerations. Neurosurgery 2010; 66: 825-31.

9. Alawieh A, Vargas J, Turner RD, et al. Equivalent favorable outcomes possible after thrombectomy for posterior circulation large vessel occlusion compared with the anterior circulation: the MUSC experience. J Neurointerv Surg 2018; 10: 735-40.

10. Widimsky P, Koznar B, Peisker T, et al. Feasibility and safety of direct catheter-based thrombectomy in the treatment of acute ischaemic stroke. Cooperation among cardiologists, neurologists and radiologists. Prospective registry PRAGUE-16. EuroIntervention 2017; 13: 131-6.
11. Ma L, Liu YH, Feng $H$, et al. Endovascular recanalization for symptomatic subacute and chronic intracranial large artery occlusion of the anterior circulation: initial experience and technical considerations. Neuroradiology 2019; 61: 833-42.

12. Jiang WJ, Cheng-Ching E, Abou-Chebl A, et al. Multicenter analysis of stenting in symptomatic intracranial atherosclerosis. Neurosurgery 2012; 70: 25-30.

13. Akif Cakar M, Tatli E, Tokatli A, et al. Percutaneous endovascular therapy for symptomatic chronic total occlusion of the left subclavian artery. Singapore Med J 2018; 59: 534-8.

Received: 19.02.2020, accepted: 14.06.2020. 